\title{
Submicrometric Heavily Doped $n$-GaAs Structures for Microwave Detection
}

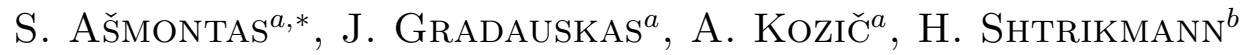 \\ AND A. SUŽIEDE்LIS ${ }^{a}$ \\ ${ }^{a}$ Semiconductor Physics Institute, A. Goštauto 11, 01108 Vilnius, Lithuania \\ ${ }^{b}$ Braun Center for Submicron Research, Weizmann Institute of Science \\ Rehovot 76100, Israel
}

\begin{abstract}
Strong coupling between electrons and phonons in heavily doped semiconductors impedes, in general, investigation of hot carrier phenomena in the material. Investigations of hot electron electromotive force arising in symmetrically and asymmetrically shaped structures of heavily doped $n$-GaAs under microwave radiation are presented in this paper. Mesas of MBE grown $n$-GaAs layers with neck shaped down to submicron dimensions revealed strong dependence of voltage sensitivity of the structure on the size of the neck. Slight frequency dependence of voltage sensitivity of the microwave diodes with both symmetrically and asymmetrically shaped $n-n^{+}$junctions was observed experimentally in $K_{a}$ frequency range, which coincides well with theoretical predictions.
\end{abstract}

PACS numbers: 84.40.--x, 07.57.Kp

\section{Introduction}

A detector of electromagnetic radiation can operate on free carrier heating effects in non-uniform semiconductor structures [1]. Planar design of asymmetrically shaped semiconductor structure with $n-n^{+}$junction in its narrowest part was used to detect microwaves, terahertz and infrared radiation [2]. The most outstanding features of the planar asymmetrically shaped detector of electromagnetic radiation were recognised as: wide frequency band of operation, high speed of operation, wide dynamic range, substantial electrical robustness. A direct (homodyne) microwave signal detection technique can be implemented using the asymmetrically shaped microwave diodes. Voltage sensitivity of the detector depends on the size of the neck of the diode [3]: reducing the width of the neck results in a higher value of the sensitivity, however, the electrical resistance of the diode increases at the same time, thus leading to the decrease of both operational speed and amount of absorbed microwave power which in turn, reduces the voltage sensitivity of the

*corresponding author; e-mail: as@uj.pfi.lt 
diode. The electrical resistance of the detector can be lowered by higher doping of the semiconductor layer. However, a certain risk of veiling of the carrier heating phenomena in heavily doped semiconductor does exist due to strong coupling between electrons and phonons in degenerate semiconductors. Nevertheless, our recent experiments with GaAs tunnel junctions evidenced the carrier heating by microwave and infrared radiation in degenerate semiconductor [4]. Here we present new experimental findings of electron heating by microwaves in heavily doped submicrometric semiconductor structures.

\section{Samples}

MBE grown structures (see Fig. 1) were used for the fabrication of microwave diodes of two different configurations: asymmetrically shaped diodes (AD) and microwave diodes with symmetrically necked semiconductor layer (SD). The height of the MBE grown $n^{+}$-GaAs layer was $h=100 \mathrm{~nm}$, while, the width $d$ of the neck of the diodes varied from $1 \mu \mathrm{m}$ up to $3 \mu \mathrm{m}$. Donor density in the $n^{+}$-GaAs layer was $N_{d}=1 \times 10^{18} \mathrm{~cm}^{-3}$. The shape of mesa of the diodes was formed using wet etching technique. Second photolithography process was done for the formation of the patterns of metallic contacts. $\mathrm{Ni} / \mathrm{Au} / \mathrm{Ge} / \mathrm{Ni} / \mathrm{Au}$ layers were evaporated thermally in the vacuum of approximately $3 \times 10^{-6}$ Torr. The pattern of metal contacts was formed by lift-off technique. Rapid annealing of
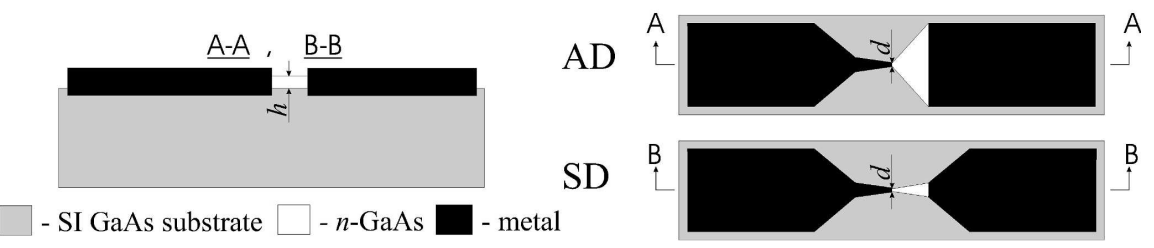

Fig. 1. Schematic view of asymmetrically (AD) and symmetrically (SD) shaped microwave diodes.

the evaporated metal contacts was performed at $430^{\circ} \mathrm{C}$ for 40 seconds in forming gas atmosphere. Quality of ohmic contacts was controlled by transmission line method. Hall measurements gave the value of electron mobility in the epitaxial layer $\mu=3000 \mathrm{~cm}^{2} \cdot \mathrm{V}^{-1} \cdot \mathrm{s}^{-1}$. The thickness of non-depleted part of epitaxial layer was calculated from the values of electrical resistances of the test structures and was found to be $30 \mathrm{~nm}$. Finally, the microwave diodes were mounted into rectangular waveguides, and the microwave measurements were performed using pulse modulated magnetron generator operating at $f=10 \mathrm{GHz}$ frequency and klystron generator in $26 \div 37 \mathrm{GHz}$ frequency range.

\section{Results and discussion}

Testing of the planar microwave diodes in dc regime showed linear currentvoltage characteristics, as well as correspondence of the electrical resistance value 
to their geometry. The polarity of the detected voltage in microwaves corresponded to that of thermoelectric effect of hot carrier in $n-n^{+}$junction. Dependences of the detected voltage on microwave power of the symmetrical diodes are presented in Fig. 2a. The voltage-power characteristics are linear in warm electron region, i.e. when the microwave electric field in the neck of the diode does not change the carrier mobility. A higher value of voltage sensitivity have the diodes with narrower neck of the structure. This confirms our theoretical estimations concerning the reciprocal dependence of the voltage sensitivity on the width of the neck [1]. However, the supperlinearity of the voltage-power characteristic was observed at a higher microwave power. We associate this supper-linear voltage-power characteristic with the rise of intervalley electromotive force in GaAs [5] as well as with increase of the electron energy relaxation time at room temperature in GaAs [6]. One more feature of presented microwave diodes should be observed. It is about non-monotonic character of the voltage-power characteristic: increase of the microwave power caused a sudden decrease of the detected voltage followed by the change of its polarity. We explain this phenomenon with the origination of negative differential resistance in $n$-GaAs layer due to the Gunn effect [5].
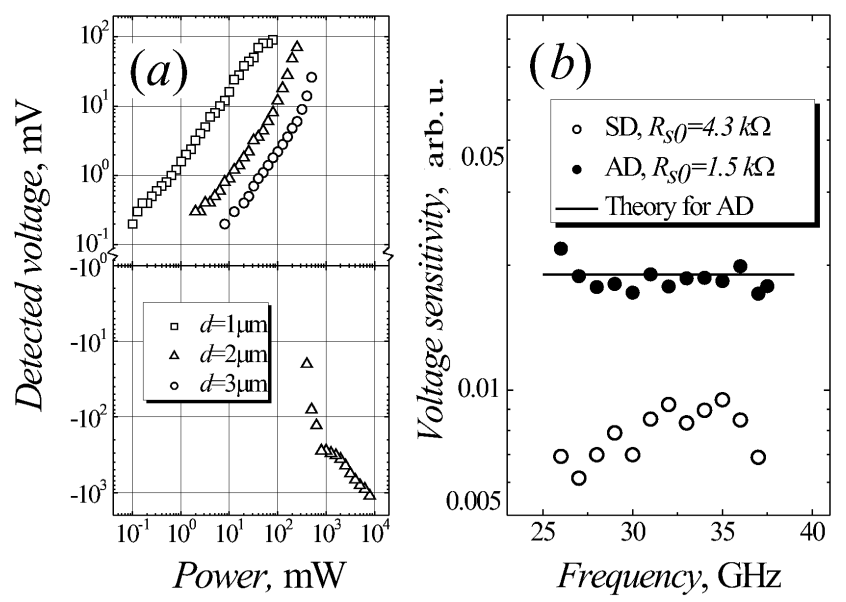

Fig. 2. (a) Voltage-power characteristics of symmetrically shaped microwave diodes with different widths of the neck and (b) frequency dependence of the asymmetrical and symmetrical microwave diodes in $K_{a}$ frequency range.

Measurements in $K_{a}$ frequency range revealed higher voltage sensitivity of the microwave diodes with asymmetrically shaped structure. Experimental frequency dependences of the voltage sensitivity of the SD and AD with the width of the neck $d=3 \mu \mathrm{m}$ are presented in Fig. 2b. Higher voltage sensitivity of the AD can be explained by a greater amount of absorbed microwave power due to their lower electrical resistance. One more reason for higher sensitivity of the AD lies in additional input of bigradient electromotive force (emf) of the asymmetrically 
shaped semiconductor structure [1] to the detected voltage, because in case of heavily doped semiconductor the polarities of thermoelectric and bigradient emfs have the same sign. It is worth to note the weak frequency dependence of the voltage sensitivity of both types of the diodes in the investigated frequency range. Solid line in Fig. 2b depicts theoretical frequency dependence of the voltage sensitivity of the microwave diode with asymmetrically shaped epitaxial layer [2]. Good agreement between calculated and experimental results is achieved when the value of $0.5 \%$ of absorbed microwave power by the microwave diode is assumed.

\section{Conclusions}

Investigation of the planar microwave diodes on the base of thin heavily doped MBE grown epitaxial layers of different shape let us to conclude:

(i) Thermoelectric electromotive force arises in heavily doped semiconductor structures which evidences the carrier heating phenomenon in degenerate semiconductor;

(ii) The voltage sensitivity of the necked semiconductor structure increases with narrowing the neck of the diode;

(iii) Thermoelectric nature of the detected voltage determines the weak frequency dependence of voltage sensitivity of the diodes in investigated frequency range.

\section{Acknowledgments}

This work was supported in part by the NATO Science for Peace Programme under the project No. 978030 and Lithuanian State Science and Studies Foundation under the project No. B-03005. Samples were fabricated at Braun Submicron Research Center under the contract No. HPRI-CT-2001-00114.

\section{References}

[1] S. Ašmontas, A. Sužiedèlis, J. Thermoelectricity N 1, 5 (1997).

[2] A. Sužiedèlis, J. Gradauskas, S. Ašmontas, G. Valušis, H.G. Roskos, J. Appl. Phys. 93, 3034 (2003).

[3] S. Ašmontas, J. Gradauskas, A. Sužiedèlis, G. Valušis, Microelectronic Engineering 53, 553 (2000).

[4] S. Ašmontas, J. Gradauskas, J. Paukštè, V. Petkun, D. Seliuta, A. Sužiedèlis, E. Širmulis, G. Valušis, Lithuanian J. Phys. 44, 263 (2004).

[5] S. Ašmontas, J. Gradauskas, J. Kundrotas, A. Sužiedèlis, A. Šilènas, G. Valušis, in: Proc. 24th Int. Conf. on Physics of Semiconductors 24ICPS'98, August 1998, Jerusalem (Israel), Ed. D. Gershoni, CD-ROM (1193.pdf), World Scientific, 1999.

[6] K. Ashida, M. Inoue, J. Shirafuji, Y. Inuishi, J. Phys. Soc. Jpn. 37, 408 (1974). 